\title{
Biologically active diterpenes containing a gem- dimethylcyclopropane subunit: an intriguing source of PKC modulators $†$
}

\author{
María Jesús Durán-Peña, José Manuel Botubol Ares, Isidro G. Collado* \\ and Rosario Hernández-Galán*
}

Covering: 1973 to 2013

Received 22nd January 2014

The biological activities of tigliane, lathyrane, ingenane, casbane, jatropholane and premyrsinane diterpenoids which contain the gem-dimethylcyclopropyl unit are described. Particular attention is given to their anti-viral, anti-microbial and cytotoxic activities. In the main text there are 132 references. The electronic supplementary information contains tables listing 424 of these diterpenoids, their occurrence

DOI: $10.1039 / \mathrm{c} 4 \mathrm{np} 00008 \mathrm{k}$ and biological activity together with the references.

www.rsc.org/npr
2.4.1 Antimicrobial activity
2.4.2 Cytotoxic activity
2.4.3 Other activities
2.5 Jatropholanes $\dagger$
2.5.1 Antimicrobial activity
2.5.2 Cytotoxic activity
2.5.3 Gastroprotective activity
2.6 Premyrsinanes $\dagger$
2.6.1 Modulation of multidrug resistance
2.6.2 Neuroprotective activity
2.7 Euphoractine group $\dagger$
2.8 Other diterpenes $\dagger$
2.8.1 Antimicrobial activity
2.8.2 Cytotoxic activity
3 Conclusions and future outlook
4 Abbreviations
5 Acknowledgements
6 References

\section{Introduction}

The study of the biological substrate-target interactions of proteins with organic molecules of low molecular weight, including Natural Products (NPs), has led to the identification of the role of these proteins in several important biological processes. This field of research has attracted great interest in recent years because it facilitates the study of complex cellular mechanisms, many of which have led to the recognition and identification of important biological targets. ${ }^{1}$ 
The Natural Products include those containing a gem-dimethylcyclopropane subunit, some of which are considered to be of great interest for the discovery of new NP-based drugs due to their wide range of biological activities and structural diversity. Some of these compounds are currently under study in preclinical or clinical trials or have already been approved for the treatment of certain diseases. ${ }^{2}$

This three-membered carbocycle is found in various types of compounds, particularly terpenoids, and these have been isolated from a large variety of organisms like plants, some fungi and marine organisms such as soft corals and insects.

Natural Products with a gem-dimethylcyclopropane unit are mainly terpenoids and this carbocycle forms part of their basic skeleton.

Tables listing the known natural products of these structural types, the species from which they were isolated, their biological activities and references are available in the Electronic Supplementary Information (ESI). $\dagger$

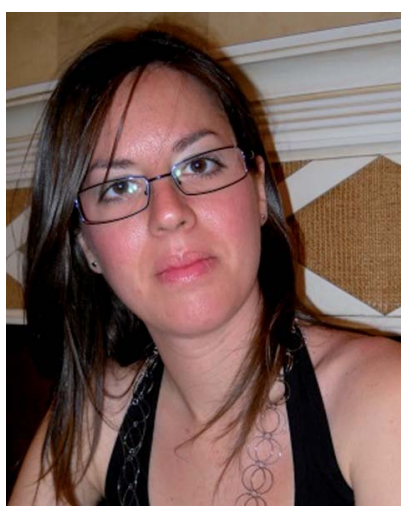

María Jesús Durán-Peña was born in 1984 in Cadiz, Spain. She studied chemistry at the University of Cádiz. She carried out her PhD at Cádiz University with Professor Dr R. H. Galán and Professor Dr I. G. Collado. Her work focuses on the isolation of secondary metabolites from euphorbia species and the development of new methodologies for cyclopropanation.

\section{Diterpenoids with a gem- dimethylcyclopropyl unit}

A large number of diterpenes contain a gem-dimethylcyclopropane ring. These diterpenes have a wide variety of carbon skeleta, derived from the cation casbane. They include tiglianes, lathyranes, ingenanes, casbanes, premyrsinanes and jatropholanes. They are mostly present in species of the Euphorbiaceae. These plants are of great interest in the search for new drugs based on Natural Products because of the wide variety of pharmacological properties they possess and the great diversity of compounds with high structural variability that they produce. ${ }^{1,3}$

These polycyclic diterpenes, occurring widely in the Euphorbiaceae, can be formed by intramolecular cyclization of casbane. ${ }^{4}$ Despite the recent advances in the understanding of the biosynthesis of many types of natural products, the specific enzymes responsible for building this strained three membered ring have only been identified in a few cases and many mechanistic details remain unclear. ${ }^{5}$

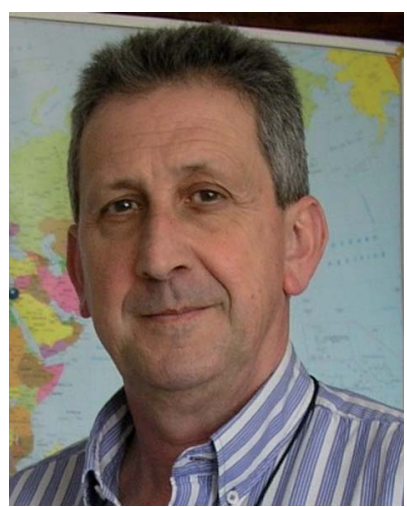

Isidro G. Collado is a Professor of Organic Chemistry at the University of Cadiz, Spain. He received a BSc in Chemistry from the University of Seville in 1978. He completed his PhD on natural product chemistry under the tutelage of Prof. Dr G. M. Massanet. He then worked as a postdoctoral fellow with Professors B. M. Fraga and J. R. Hanson on gibberellin chemistry at the Consejo Superior de Investigaciones Cientificas (Tenerife) and Sussex University, respectively. His current research interests include the genomic bases of natural product biosynthesis, synthesis of bioactive molecules and design of selective fungicides.

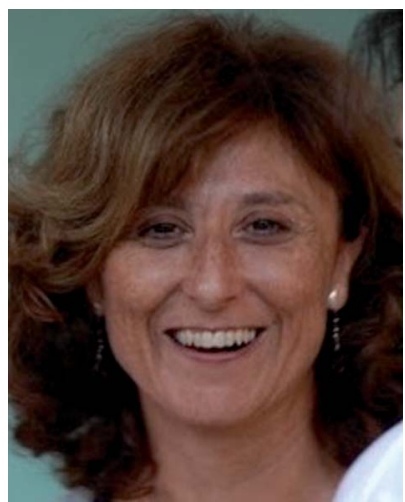

R. Hernández-Galán is a Professor of Organic Chemistry at the University of Cádiz, Spain. She obtained her PhD degrees from the University of Cádiz in 1991 for her work on the synthesis of 3-(1,1-dimethylallyl)coumarins under the tutelage of Professor F. R. Luis. She completed her PhD studies at the Dyson Perrins Laboratory, Oxford with Professor Laurence M. Harwood. Her current research interests include the biosynthesis of natural products, synthesis of bioactive compounds and the design and synthesis of agonists of PKCs. 
A number of enzymes in the terpenoid synthase family that catalyze cyclopropane formation have also been biochemically and mechanistically characterized recently, especially those responsible for the production of the bicyclic plant monoterpenes such as carene or sabinene synthase. ${ }^{5}$ However the reported data on the biosynthesis and enzymatic formation of the cyclopropane ring in diterpenes are sparse. At least one diterpene synthase, casbene synthase, has been shown to cyclize geranylgeranyl pyrophosphate (GGPP) into casbene, which seems to be the key biosynthetic precursor of the lathyrane skeleton, an intermediate to jatropholane, tigliane, or premyrsinane which retain the cyclopropane ring in their final structures..$^{5-9}$

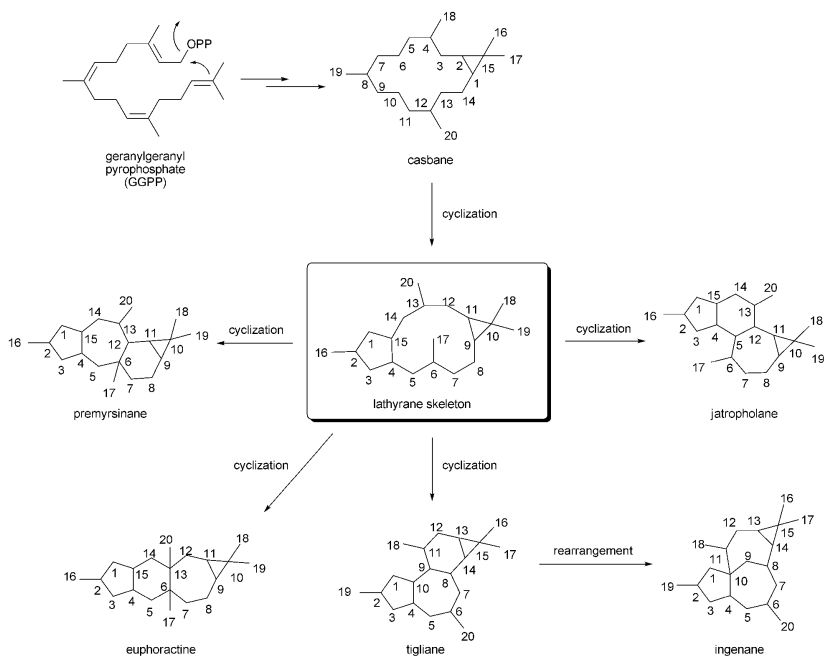

This enzyme seems to follow a mechanism where cleavage of the allylic pyrophosphate moiety of GGPP, by a divalent metal ion, leads to an allylic carbocation that through alkylation and deprotonation generates the cyclopropane moiety in the casbane skeleton..$^{6-9}$

The ring strain present in the gem-dimethylcyclopropane moiety looks to be responsible for the biological activities of these compounds, many of them are potent alkylation agents. However, few reports about the biological relevance of this subunit have been published. For more detailed information about the biosynthesis and biological activity of this interesting subunit, the reader is directed to a recent excellent review. ${ }^{5}$

Tiglianes and ingenanes, together with daphnanes are referred to as phorboids and are known for being proinflammatory agents and tumor promoters which behave as protein kinase C (PKC) activators. Proof of the pharmacological relevance

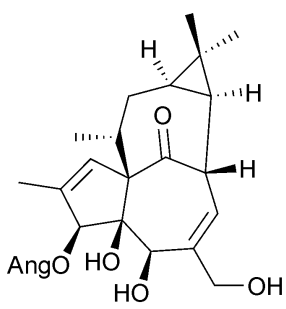

(1) of this group of compounds is the FDA's approval in January 2012 of 3-O-angeloylingenol (PEP-005, Picato ${ }^{\circledR}$, Leo Pharma) for the treatment of actinic keratosis, a precancerous skin condition. ${ }^{1}$

\subsection{Tiglianes $\dagger$}

Protein Kinase Cs (PKCs) are involved in many physiological functions and certain NPs, such as phorbol esters, have proved to be important activators of these proteins. Certain phorbol esters can activate PKCs, but after an incubation period they decrease its regulatory activity. The activating capacity of PKCs is responsible for the wide variety of biological activities reported for this group of compounds such as platelet aggregation, ${ }^{10}$ cell differentiation, ${ }^{11,12}$ metabolic activity, ${ }^{13-15}$ irritant, ${ }^{16}$ tumor-promotion, ${ }^{17,18}$ anti-HIV-1, ${ }^{19,20}$ cytotoxicity ${ }^{21}$ and as a molluscicide. ${ }^{22}$

2.1.1 Anti-HIV activity. 12-Deoxyphorbols such as prostratin (13-O-acetyl-12-deoxyphorbol (2)) are a subclass of PKC activators since, unlike other phorbol esters, these do not induce tumor formation. Prostratin (2) has been found in Euphorbia species. ${ }^{23,24}$ Recently, it has been shown that prostratin is a PKC activator that is potentially useful in the treatment of HIV as it affects viral reservoirs in $\mathrm{CD}^{+}{ }^{+}$T-cells with a latent infection. ${ }^{25}$ DPP (3) induces HIV-1 gene expression in latently infected cells at concentrations 20-40 times lower than prostratin. ${ }^{26}$ Considering that the only structural difference between prostratin (2) and DPP (3) is the nature of the ester group bonded to the $\mathrm{C}-13$ carbon, it is easy to associate the activity of the 12-deoxyphorbol skeleton with other phorbol esters such as 13-O-benzoyl-12-deoxyphorbol (4) which have exhibited the same type of behaviour in other studies. ${ }^{27,28}$ Epimers 5 and 6 are potent PKC activators. ${ }^{29}$ Fischerosides A-C
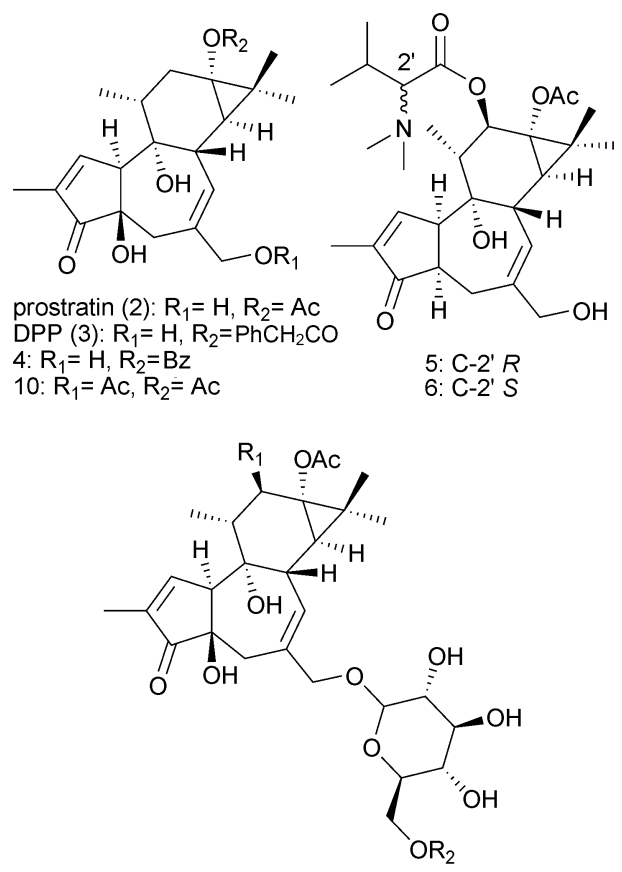

fischeroside $A$ (7): $R_{1}=H, R_{2}=H$

fischeroside $B(8): R_{1}=H, R_{2}=$ galloyl

fischeroside $\mathrm{C}(9): \mathrm{R}_{1}=\mathrm{OH}, \mathrm{R}_{2}=\mathrm{H}$ 
(7-9), tiglianes that carry a glycoside group on the C-20 carbon, have a mild inhibitory effect on the HIV-1 virus. Fischeroside C (9) had an $\mathrm{EC}_{50}$ value of $0.02 \mu \mathrm{M}$ and a therapeutic index of 17.50, while prostratin (2) and 13,20-O-diacetyl-12-deoxyphorbol (10) were significantly more active. ${ }^{30}$

2.1.2 Cytotoxic activity. Important protein kinase C (PKC) activators, which show great potential as HIV-1 activators, were isolated from different species of the Euphorbia genus. PMA (or TPA, 11) is used extensively as a molecular biological research tool. It is known to stimulate the kinase activity of PKC and antagonize HIV-1 latency efficiently. ${ }^{17,31,32}$ Compounds 12-19 displayed cytotoxicity on human cancer cell lines. ${ }^{33-36}$ Euphodendriane A (20) and pedilstatin (21) are cancer cell growth inhibitors. $^{37,38}$

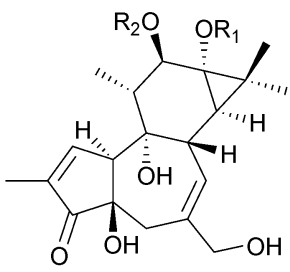

PMA (11): $\mathrm{R}_{1}=\mathrm{Ac}, \mathrm{R}_{2}=\mathrm{CO}\left(\mathrm{CH}_{2}\right)_{12} \mathrm{CH}_{3}$ 12: $\mathrm{R}_{1}=\mathrm{CO}\left(\mathrm{CH}_{2}\right)_{8} \mathrm{CH}_{3}, \mathrm{R}_{2}=\mathrm{Tig}$
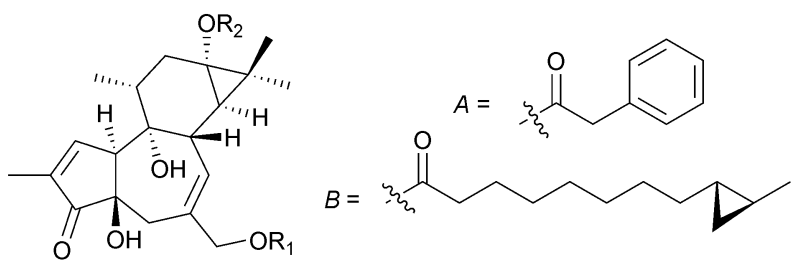

$$
\begin{aligned}
& \text { 13: } R_{1}=A c, R_{2}=A n g \\
& \text { 14: } R_{1}=A c, R_{2}=A \\
& \text { 15: } R_{1}=H, R_{2}=B \\
& \text { 16: } R_{1}=H, R_{2}=O C\left(C_{2}\right)_{14} M e \\
& \text { 17: } R_{1}=\text { decanoyl, } R_{2}=\text { octanoyl } \\
& \text { 18: } R_{1}=\text { decanoyl, } R_{2}=\text { decanoyl } \\
& \text { 19: } R_{1}=\text { decanoyl, } R_{2}=\text { dodecanoyl }
\end{aligned}
$$

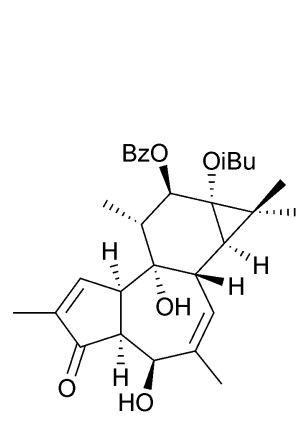

euphodendriane $A$

(20)

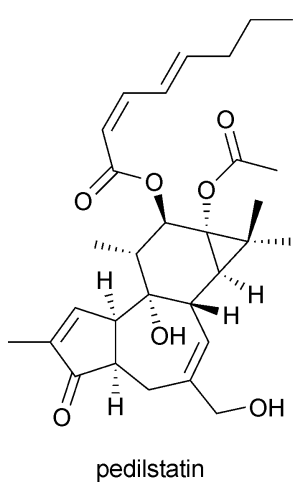

(21)
2.1.3 Inhibitory activity on the mitochondrial respiratory chain. Compounds 22-25 were active when evaluated for their inhibition of $\mathrm{NADH}$ oxidase activity in submitochondrial particles from beef heart. All of them are described as NADH oxidase inhibitors, 22 being the strongest inhibitor of NADH oxidase activity with an $\mathrm{IC}_{50}$ value of $2.60 \mathrm{mM} .^{39}$

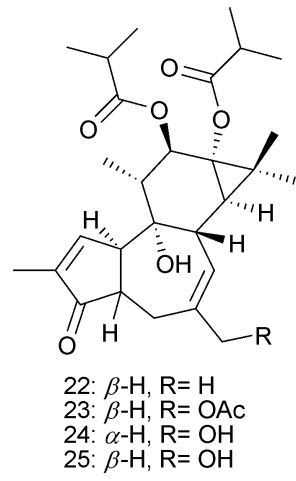

2.1.4 Toxic activity. It is known that ingestion of certain members of the Euphorbiaceae by animals reduces their performance, contaminates milk, decreases reproduction and can even cause death. ${ }^{40}$ This toxicity is associated with phorbol esters such as PMA (11). The phorbols 26-33 are known to be toxic and are also powerful irritants. ${ }^{41}$ Jatropherol-I (34) was highly toxic to silkworm larvae, ${ }^{\mathbf{4 2 , 4 3}}$ moreover compounds 35-51 acted as irritants. ${ }^{4-50}$ On the other hand, compounds 52-59 exhibit

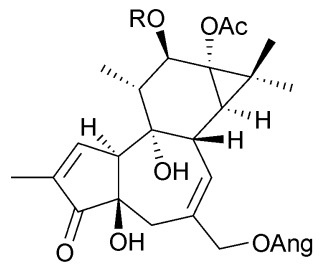

26: $R=i B u$

43: $\mathrm{R}=\mathrm{COCHMe}$

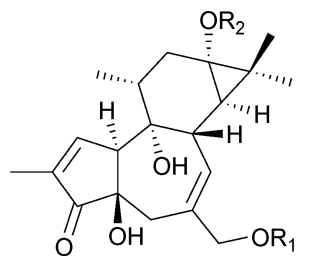

27: $\mathrm{R}_{1}=\mathrm{H}, \mathrm{R}_{2}=\alpha-\mathrm{MeBu}$

28: $\mathrm{R}_{1}=\mathrm{H}, \mathrm{R}_{2}=$ heptanoy

29: $\mathrm{R}_{1}=\mathrm{H}, \mathrm{R}_{2}=$ lauroyl

40: $R_{1}=A c, R_{2}=\alpha$-MeBu

41: $R_{1}=A c, R_{2}=$ lauroyl

52: $\mathrm{R}_{1}=\mathrm{Ang}, \mathrm{R}_{2}=\mathrm{Ac}$

53: $\mathrm{R}_{1}=A c, \mathrm{R}_{2}=D$<smiles>Nc1ccccc1C(=O)Nc1ccccc1C(=O)O</smiles>

54: $R_{1}=B z, R_{2}=B z$

55: $\mathrm{R}_{1}=$ Ang, $\mathrm{R}_{2}=$ Ang

56: $\mathrm{R}_{1}=D, \mathrm{R}_{2}=$ Ang

57: $\mathrm{R}_{1}=D, \mathrm{R}_{2}=$ Tig

58: $\mathrm{R}_{1}=D, \mathrm{R}_{2}=\mathrm{Bz}$

59: $\mathrm{R}_{1}=D, \mathrm{R}_{2}=$ hexanoyl

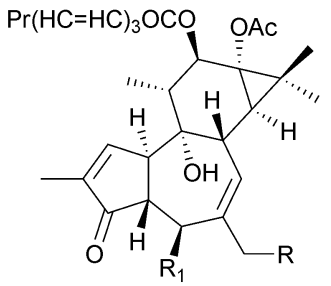

sapatoxin A (30): $\mathrm{R}=\mathrm{OH}, \mathrm{R}_{1}=\mathrm{H}$ sapatoxin $B$ (31): $R=R_{1}=O H$ sapatoxin $C(32): R=H, R_{1}=O H$

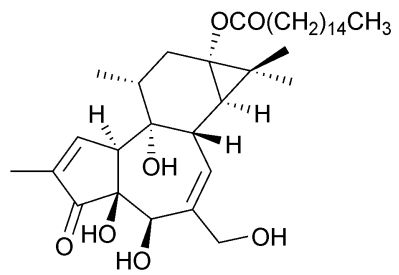

stillingia factor S8

(33) 
molluscicidal activity against the snail, Biomphalaria glabrata. This snail is the major intermediate host for Schistosoma mansoni, one of the important schistosomes infecting man..$^{21,51}$

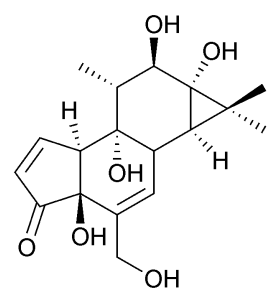

jatropherol-I

(34)

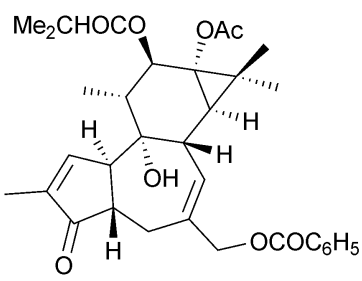

38

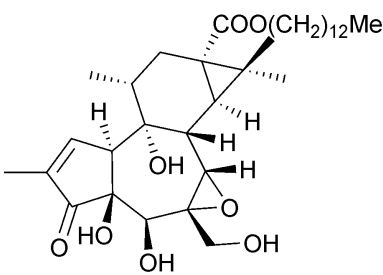

42

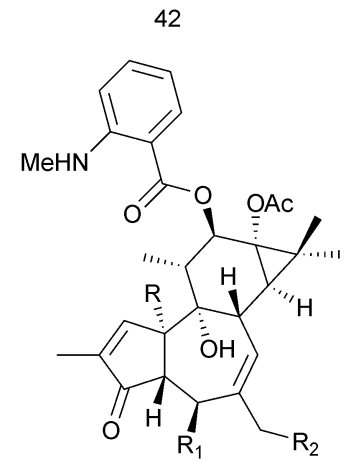

sapintoxin A (47): $\mathrm{R}=\mathrm{R}_{1}=\mathrm{H}, \mathrm{R}_{2}=\mathrm{OH}$ sapintoxin $B(48): R=H, R_{1}=R_{2}=O H$ sapintoxin $\mathrm{C}$ (49): $\mathrm{R}=\mathrm{R}_{2}=\mathrm{H}, \mathrm{R}_{1}=\mathrm{OH}$ sapintoxin D (50): $\mathrm{R}=\mathrm{R}_{2}=\mathrm{OH}, \mathrm{R}_{1}=\mathrm{H}$

2.1.5 Tumor promotion activity. PMA (11) is a known protein kinase $\mathrm{C}$ activator that promotes tumors. ${ }^{52-54}$ The esters, 35-39 also display tumor-promoting activity. ${ }^{47}$ Phorbol esters do not induce tumors on their own but rather promote their growth by continuous exposure to certain levels of these esters. ${ }^{38}$

In contrast to this ability to activate tumor growth, many malignant cells have been observed entering apoptosis in response to PKC activation by phorbol esters. PKC activity displacement is attributed to its role in the activation of different metabolic pathways, its cellular localization, phosphorylation, interaction with other molecules and accessibility to various substrates. ${ }^{55,56}$

2.1.6 Other activities. Prostratin (2) exhibited analgesic and sedative effects in preliminary pharmacological assays carried out with mice. ${ }^{22}$ Fischeroside B (8) provided significant protection against the oxidative stress caused by tert-butylhydroperoxide by inhibiting the generation of reactive oxygen species and increasing glutathione levels in HepG2 cells. ${ }^{57}$ Trigowiin A (60) exhibited antiviral activity against the Chikungunya virus. ${ }^{58}$

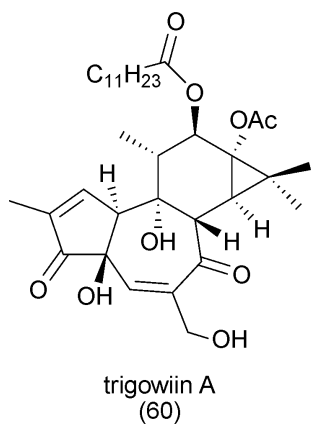

\subsection{Lathyranes $\dagger$}

2.2.1 Antimicrobial activity. Diterpenes 61-63 exhibited antibacterial activity against Bacillus subtilis and Staphylococcus aureus. ${ }^{5-61}$

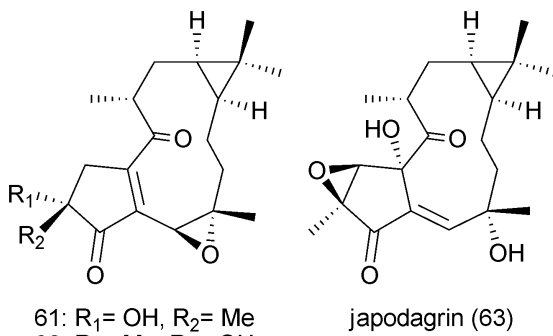

2.2.2 Antiviral activity. Latilagascenes A-C (64-66), E (67) and jolkinol B (68) decreased immediate-early (IE) antigen expression of the human cytomegalovirus (CMV). These antiviral compounds also act as anti-tumour compounds towards certain forms of cancers that are associated with human CMV. ${ }^{62}$ On the other hand, the HIV/AIDS field is gaining momentum with the goal of finding a functional cure for HIV infection by utilizing strategies that specifically reactivate the latent viral reservoir in combination with the Highly Active Antiretroviral Therapy (HAART). The virus HIV can be destroyed by HAART when it is active. ${ }^{63}$ Compounds 69 and 70 were able to reactivate HIV-1 latency. Results with 69 strongly suggest that it reactivates

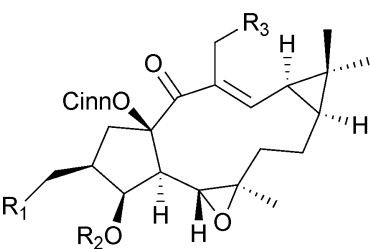

64: $R_{1}=O A c, R_{2}=R_{3}=H$ 65: $\mathrm{R}_{1}=\mathrm{OH}, \mathrm{R}_{2}=\mathrm{R}_{3}=\mathrm{H}$ 66: $R_{1}=O A C, R_{2}=A C, R_{3}=H$ 67: $\mathrm{R}_{1}=\mathrm{OBz}, \mathrm{R}_{2}=\mathrm{H}, \mathrm{R}_{3}=\mathrm{OH}$ jolkinol $B(68)$ : $R_{1}=R_{2}=R_{3}=H$

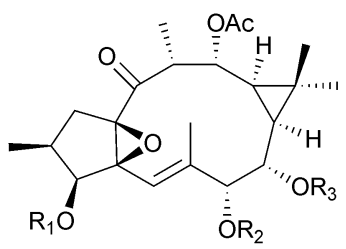

69: $R_{1}=A c, R_{2}=H, R_{3}=$ Tig 70: $R_{1}=B z, R_{2}=A c, R_{3}=M e$ 
HIV-1 through a PKC-dependent pathway. ${ }^{30}$ Compound $\mathbf{7 0}$ induced cell-cycle arrest and HIV-1-LTR promoter activation and could be a novel lead compound for the development of therapies against HIV-1 latency. ${ }^{64}$

2.2.3 Cytotoxic activity. Latilagascene B (65) showed antitumour activity against gastric carcinoma cells. ${ }^{65}$ The diterpenoid esters 71-80 exhibited important cytotoxic activity. ${ }^{66,67}$ Euphorbia factors $\mathrm{L}_{1}(\mathbf{8 1}), \mathrm{L}_{3}(\mathbf{8 2})$ and japodagrol (83) exhibited in vitro anticancer activity. ${ }^{68,69}$

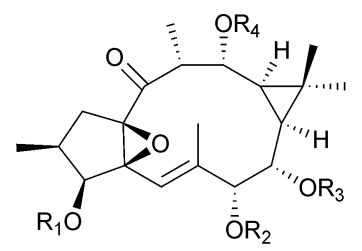

71: $R_{1}=2-M e B u, R_{2}=R_{3}=R_{4}=A C$ 72: $R_{1}=R_{3}=R_{4}=A c, R_{2}=2-M e B u$ 73: $R_{1}=R_{2}=R_{4}=A c, R_{3}=2-M e B u$ 74: $R_{1}=R_{2}=R_{3}=A c, R_{4}=2-M e B u$ 75: $R_{1}=2-M e B u, R_{2}=R_{4}=A c, R_{3}=M e$ 76: $R_{1}=R_{4}=A c, R_{2}=2-M e B u, R_{3}=M e$ 77: $R_{1}=R_{2}=A c, R_{3}=M e, R_{4}=2-M e B u$ 78: $R_{1}=R_{4}=A c, R_{2}=A n g, R_{3}=M e$ 79: $R_{1}=H, R_{2}=A n g, R_{3}=M e, R_{4}=A c$ 80: $R_{1}=R_{4}=A c, R_{2}=H, R_{3}=M e$

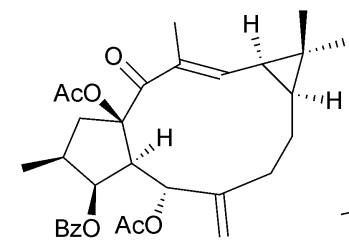
euphorbia factor $L_{3}$ (82)

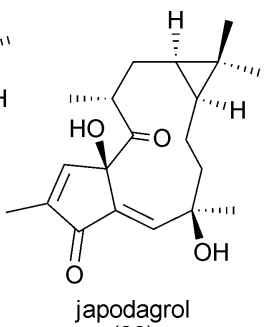

(83)

2.2.4 Modulation of multidrug resistance. P-Glycoprotein (P-gp) is a multidrug transporter which is overexpressed in cancer cell plasma membranes as an efflux pump conferring cellular resistance to anticancer chemotherapy. Latilagascene B (65) was found to be an effective P-gp inhibitor and it is an inhibitor of multidrug resistance by tumor cells. It proved highly efficient in inhibiting rhodamine 123 efflux of the human MDR1 gene from transfected mouse lymphoma cells when compared to the untreated cells or the positive control verapamil. ${ }^{70,71}$ Euphorbia factor $L_{10}(\mathbf{8 4})$ also inhibited the transport activity of this protein, exhibiting a higher level of activity than that of the specific and potent P-gp inhibitor cyclosporin A. ${ }^{72}$
2.2.5 Other activities. Latilagascenes A-C (64-66) and jolkinol B (68) are antitumor promoters and diminished the IE antigen expression of cytomegalovirus (CMV). ${ }^{60}$ Besides, latilagascenes C (66) and D (85) exhibited high antineoplastic activity. ${ }^{63}$ Lathyrane 69 has recently been patented as a promoter of stem cell proliferation in mouse brain. ${ }^{73}$ Lathyrane $\mathbf{7 9}$ was particularly active as a prostaglandin $\mathrm{E}_{2}$ inhibitor ${ }^{74}$ and lathyrane $\mathbf{8 6}$ is a potent active ingredient in rabbit basilar and carotid arteries at resting tension. ${ }^{75}$ Finally, lathyranes 87-91 showed significant vascular-relaxing activity. ${ }^{76}$

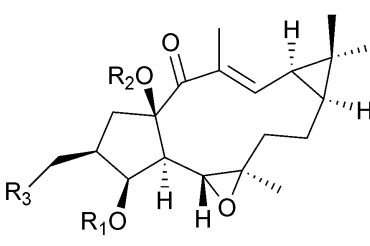

85: $\mathrm{R}_{1}=\mathrm{H}, \mathrm{R}_{2}=$ Cinn, $\mathrm{R}_{3}=\mathrm{OBz}$ 87: $R_{1}=R_{2}=B z, R_{3}=H$
88. $R_{1}=B z, R_{2}=R_{3}=H$

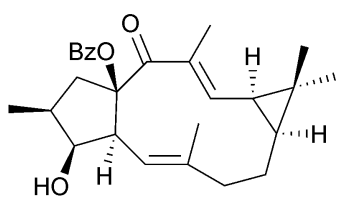
89

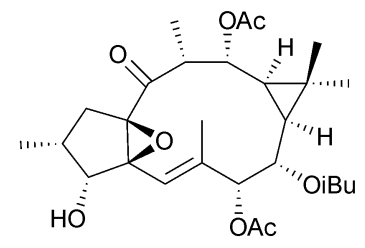

86

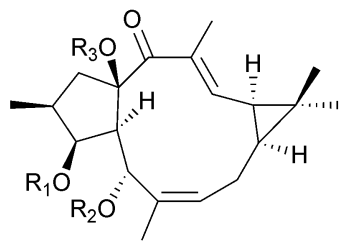

90: $\mathrm{R}_{1}=$ Cinn, $\mathrm{R}_{2}=\mathrm{R}_{3}=\mathrm{H}$ 91: $R_{1}=R_{3}=H, R_{2}=$ Cinn

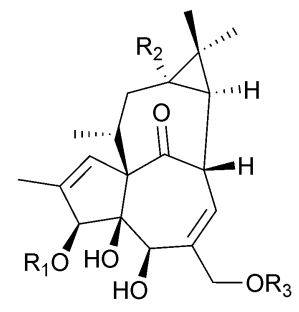

PEP-008 (92): $\mathrm{R}_{1}=$ Ang, $\mathrm{R}_{2}=\mathrm{H}, \mathrm{R}_{3}=\mathrm{AC}$ euphorbia factor $L_{5}(93): R_{1}=C O\left(C_{2}\right)_{14} C H_{3}, R_{2}=R_{3}=H$ kansuiphorin $\mathrm{A}$ (94): $\mathrm{R}_{1}=2$,3-dimethylbutanoyl, $\mathrm{R}_{2}=$ dodecanoyloxy, $\mathrm{R}_{3}=$ hexadecanoyl 98: $\mathrm{R}_{1}=\mathrm{CO}(\mathrm{CH}=\mathrm{CH})(\mathrm{CH}=\mathrm{CH})\left(\mathrm{CH}_{2}\right)_{4} \mathrm{CH}_{3}, \mathrm{R}_{2}=\mathrm{R}_{3}=\mathrm{H}$ Bz, $\mathrm{R}_{3}$ (Z)

99: $R_{1}=R_{2}=B z, R_{3}=H$

100: $R_{1}=2$,3-dimethylbutanoyl, $R_{2}=$ dodecanoyl, $R_{3}=H$ 101: $R_{1}=B z, R_{2}=$ octanoyl, $R_{3}=H$

102: $R_{1}=2$,3-dimethylbutanoyl, $R_{2}=$ octanoyl, $R_{3}=H$

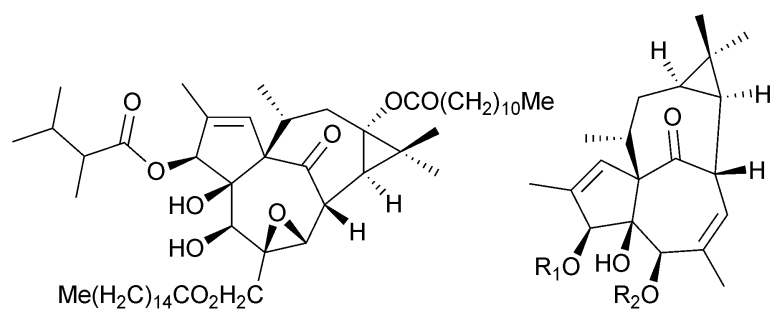

kansuiphorin B (95)

kansuiphorin $C$ (96): $R_{1}=A c, R_{2}=B z$ kansuiphorin $D(97): R_{1}=B z, R_{2}=A C$ 


\subsection{Ingenanes $\dagger$}

2.3.1 Cytotoxic activity. PEP-005 (1) is a PKC activator exhibiting topical antitumor activity against human cancer cell lines in mice and against actinic keratosis in humans. PEP-005 also appears to have antileukemic effects, inducing apoptosis in leukemia cell lines and primary acute myeloid leukemia blasts. ${ }^{77}$ The related compound PEP-008 (92) is a novel PKCactivating drug that arrests growth with senescence characteristics in solid cell lines derived from a variety of tissue types. This compound may therefore have therapeutic potential in a subset of breast cancer, colon cancer and melanoma tumors. ${ }^{78}$ Euphorbia factor $\mathrm{L}_{5}(\mathbf{9 3})$ acts as a cocarcinogen on the back skin in mice. ${ }^{79}$ Kansuiphorin A-D (94-97) and 98-102 were cytotoxic against various cancer cell lines. ${ }^{34,35,80,83}$

2.3.2 Effects on cell division. Results showed that esters 98 and 103-112 significantly inhibited cell division $\left(0.5 \mu \mathrm{g} \mathrm{ml} \mathrm{m}^{-1}\right.$ of each compound led to a reduction in cell division above $75 \%$ ). Amongst these, $\mathbf{1 1 0}$ and $\mathbf{1 1 1}$ significantly prevented cell cleavage. It should be noted that inhibitory activity was weaker in compounds with an acyl residue at position 13. Besides, compound 113 was tested and showed some activity $\left(10 \mu \mathrm{g} \mathrm{ml}^{-1}\right.$ of this compound led to a $60 \%$ reduction in cell division).$^{\mathbf{8 4 , 8 5}}$

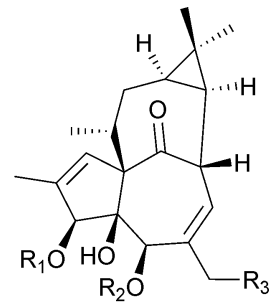

$$
\begin{aligned}
& \text { 103: } \mathrm{R}_{1}=\mathrm{CO}(\mathrm{CH}=\mathrm{CH})(\mathrm{CH}=\mathrm{CH})\left(\mathrm{CH}_{2}\right)_{4} \mathrm{CH}_{3}, \mathrm{R}_{2}=\mathrm{H}, \mathrm{R}_{3}=\mathrm{OAC} \\
& \text { (E) (Z) } \\
& \begin{aligned}
\text { 104: } \mathrm{R}_{1}=\mathrm{H}, \mathrm{R}_{2}=\mathrm{H}, \mathrm{R}_{3}=\underset{\mathrm{OCO}(\mathrm{CH}=\mathrm{CH})(\mathrm{CH}=\mathrm{CH})\left(\mathrm{CH}_{2}\right)_{4} \mathrm{Me}}{(E)} \text { 105: } \mathrm{R}_{1}=\mathrm{H}, \mathrm{R}_{2}=\mathrm{H}, \mathrm{R}_{3}=\operatorname{OCO}(\mathrm{CH}=\mathrm{CH})(\mathrm{CH}=\mathrm{CH})\left(\mathrm{CH}_{2}\right)_{4} \mathrm{Me}
\end{aligned} \\
& \text { 106: } \mathrm{R}_{1}=\mathrm{CO}(\mathrm{CH}=\mathrm{CH})(\mathrm{CH}=\mathrm{CH})\left(\mathrm{CH}_{2}\right)_{4} \mathrm{Me}, \mathrm{R}_{2}=\mathrm{COMe}, \mathrm{R}_{3}=\mathrm{OH} \\
& \text { 107: } \mathrm{R}_{1}=\mathrm{CO}(\mathrm{CH}=\mathrm{CH})(\mathrm{CH}=\mathrm{CH})\left(\mathrm{CH}_{2}\right)_{4} \mathrm{Me}, \mathrm{R}_{2}=\mathrm{H}, \mathrm{R}_{3}=\mathrm{OAC} \\
& \text { (E) (E) } \\
& \text { 108: } \mathrm{R}_{1}=\mathrm{H}, \mathrm{R}_{2}=\mathrm{H}, \mathrm{R}_{3}=\mathrm{OCO}\left(\mathrm{CH}_{2}\right)_{8} \mathrm{Me} \\
& \text { 109: } \mathrm{R}_{1}=\mathrm{H}, \mathrm{R}_{2}=\mathrm{CO}(\mathrm{CH}=\mathrm{CH})(\mathrm{CH}=\mathrm{CH})\left(\mathrm{CH}_{2}\right)_{4} \mathrm{Me}, \mathrm{R}_{3}=\mathrm{OH} \\
& \text { 110: } \mathrm{R}_{1}=\mathrm{CO}(\mathrm{CH}=\mathrm{CH})(\mathrm{CH}=\mathrm{CH})\left(\mathrm{CH}_{2}\right)_{4} \mathrm{CH}_{3}, \mathrm{R}_{2}=\mathrm{H}, \mathrm{R}_{3}=\mathrm{H} \\
& \text { 111: } \mathrm{R}_{1}=\mathrm{CO}(\mathrm{CH}=\mathrm{CH})(\mathrm{CH}=\mathrm{CH})\left(\mathrm{CH}_{2}\right)_{4} \mathrm{CH}_{3}, \mathrm{R}_{2}=\mathrm{H}, \mathrm{R}_{3}=\mathrm{H} \\
& \text { 111: } \mathrm{R}_{1}=\mathrm{CO}(\mathrm{CH}=\mathrm{CH})(\mathrm{CH}=\mathrm{CH})\left(\mathrm{CH}_{2}\right)_{4} \mathrm{CH}_{3}, \mathrm{R}_{2}=\mathrm{H}, \mathrm{R}_{3}=\mathrm{H} \\
& \text { (E) (Z) } \\
& \text { 112: } \mathrm{R}_{1}=\mathrm{CO}(\mathrm{CH}=\mathrm{CH})(\mathrm{CH}=\mathrm{CH})\left(\mathrm{CH}_{2}\right)_{4} \mathrm{CH}_{3}, \mathrm{R}_{2}=\mathrm{H}, \mathrm{R}_{3}=\mathrm{OH}
\end{aligned}
$$

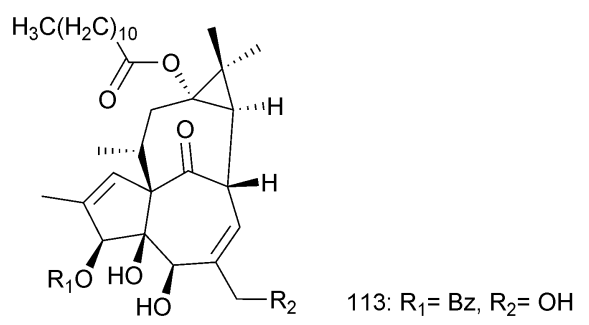

2.3.3 Irritant activity. PEP-005 (1), euphorbia factor $L_{5}(93)$ and compounds 114-118 exhibit irritant activity. ${ }^{\mathbf{8 6}-90}$
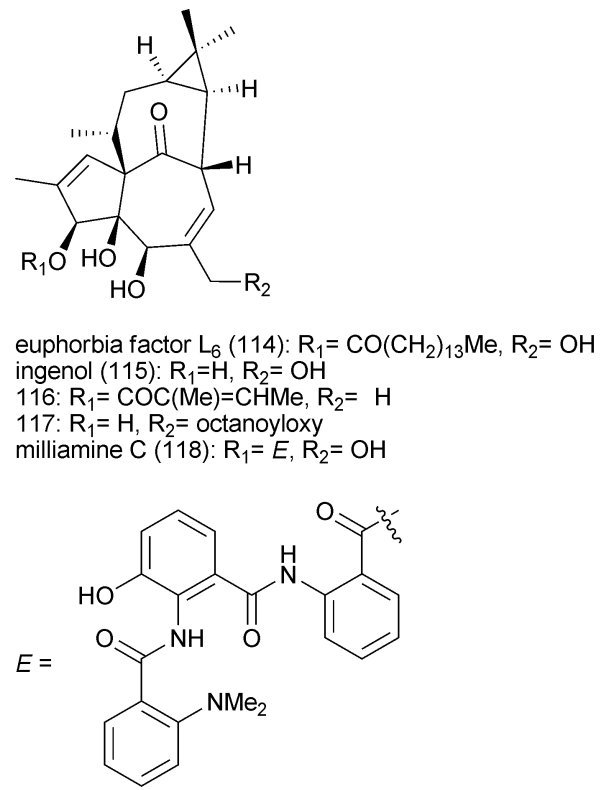

2.3.4 Pesticidal activity. Ingenol esters 112 and 119-122 exhibited antinematodal activity against the pine-wood nematode Bursaphelenchus xylophilus. This nematode is the causal agent of pine wilt disease, one of the most damaging pest problems found in forests. ${ }^{\mathbf{8 3 , 9 1 - 9 4}}$

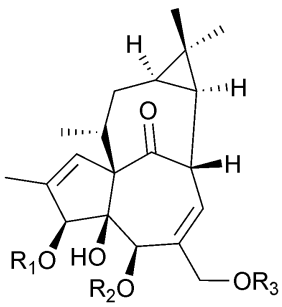

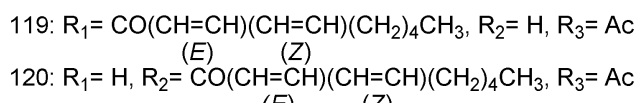

(E)

(Z)

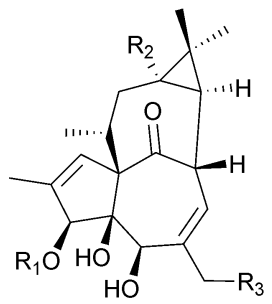

121: $\mathrm{R}_{1}=2$,3-dimethylbutanoyl, $\mathrm{R}_{2}=$ dodecanoyloxy, $\mathrm{R}_{3}=\mathrm{OH}$ DBDI (122): $R_{1}=2,3$-dimethylbutanoyl, $\mathrm{R}_{2}=$ decanoyloxy, $\mathrm{R}_{3}=\mathrm{OH}$

Compounds 123-131 exhibited molluscicidal activity. Amongst these, milliamine L (131) proved to be one of the most potent molluscicidal substances discovered to date under laboratory conditions..$^{95-97}$

Compounds 98 and 100 showed insecticidal activity. ${ }^{98}$ Results suggest that both compounds could be used directly as natural pesticides or as lead ingredients for the control of 


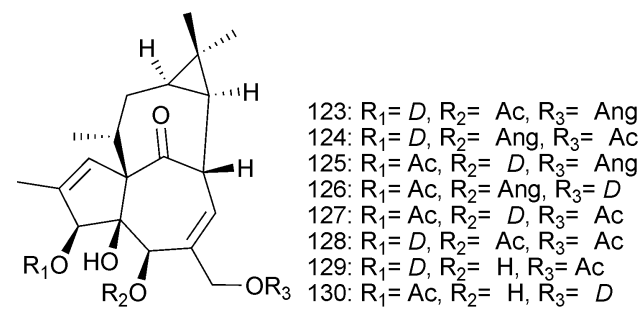<smiles>Nc1ccccc1C(=O)Nc1ccccc1C(=O)O</smiles>

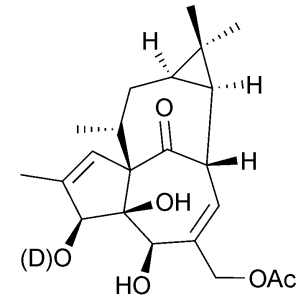

milliamine $\mathrm{L}$

(131)

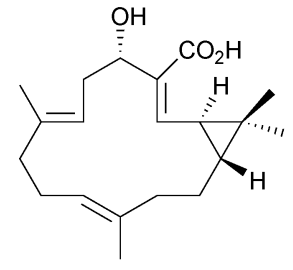

yuexiandajisu $\mathrm{A}$

(142)<smiles>C/C=C(\C)C(=O)C/C=C\C(O)C/C(C)=C/C[C@@H]1[C@@H](C)[C@H]1C=C(C)C</smiles>

10-hydroxydepressin

(143)

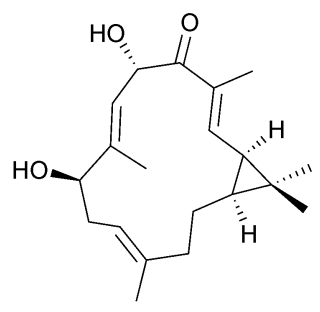

144 brown plant hopper and two-spotted spider mite. ${ }^{\mathbf{8 1}}$ Ingenol3,20-diesters 132-138 showed weak piscicidal activity. ${ }^{99}$

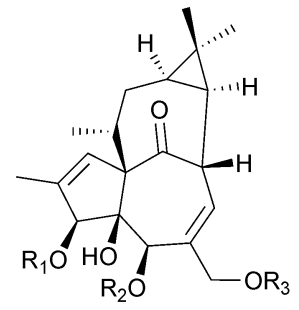

$$
\begin{aligned}
& \text { 132: } R_{1}=\text { COEt, } R_{2}=H, R_{3}=2-M e B u \\
& \text { 133: } R_{1}=H, R_{2}=H, R_{3}=C O C H M e_{2} \\
& \text { 134: } R_{1}=\text { COEt, } R_{2}=H, R_{3}=C O C H M e_{2} \\
& \text { 135: } R_{1}=\text { COCHMe }, R_{2}=H, R_{3}=C O C H M e_{2} \\
& \text { 136: } R_{1}=A c, R_{2}=A C, R_{3}=A C \\
& \text { 137: } R_{1}=H, R_{2}=H, R_{3}=2-M e B u \\
& \text { 138: } R_{1}=\text { COEt, } R_{2}=\text { COEt, } R_{3}=\text { COCHMe } \\
& \text { 139: } R_{1}=A c, R_{2}=H, R_{3}=A n g \\
& \text { 140: } R_{1}=A n g, R_{2}=H, R_{3}=A C \\
& \text { 141: } R_{1}=\text { Ang, } R_{2}=O A c, R_{3}=O A C
\end{aligned}
$$

2.3.5 Tumor-promoting activity. Biological investigations revealed that euphorbia factor $\mathrm{L}_{5}(\mathbf{9 3})$ and compounds 139 and $\mathbf{1 4 0}$ are tumor promoters. ${ }^{\mathbf{1 0 0 , 1 0 1}}$

2.3.6 Other activities. Kansuiphorin A (94) showed antiviral activity and it is suggested that this compound prevents viral pneumonia in mice. ${ }^{102}$ DBDI (122) inhibits degranulation in rat basophilic leukemia cells and results suggest that DBDI may have therapeutic potential for allergic diseases ${ }^{103}$ and finally, compound 141 exhibits antiangiogenic activity. ${ }^{\mathbf{1 0 4}}$

\subsection{Casbanest}

Diterpenes with casbane skeletons are rarely found in plants. However, some have been isolated from plants of the Euphorbia genus. ${ }^{\text {105-109 }}$

2.4.1 Antimicrobial activity. Yuexiandajisu A (142) and 10hydroxydepressin (143) exhibited antibacterial activity. ${ }^{\mathbf{1 1 0}}$ Additionally, casbene $\mathbf{1 4 4}$ had an antimicrobial effect on planktonic forms and the biofilm arising from some bacteria and yeasts. ${ }^{111}$

2.4.2 Cytotoxic activity. 10-Hydroxydepressin (143) and casbenes 145-154 were cytotoxic against cancer cell lines. ${ }^{106,109,112-115}$

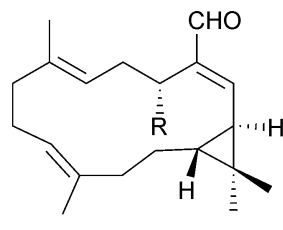

$$
\text { pekinenal (145): } \mathrm{R}=\mathrm{OH}
$$
pekinenin $F(146): R=H$<smiles>C/C(=C\[C@H]1[C@H](C)[C@H]1CC[C@@H](C)CC(=O)C1O[C@H]1C)C(=O)/C=C/[C@@]1(C)O[C@@H]1C</smiles>

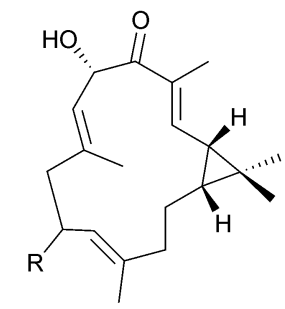

148: $R==0$ agrostistachin (149): $\mathrm{R}=\beta-\mathrm{OH}$

microclavatin (147)

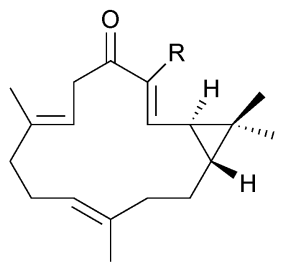

pekinenin $\mathrm{A}$ (151): $\mathrm{R}=\mathrm{CH}_{2} \mathrm{OH}$ pekinenin $\mathrm{C}$ (152): $\mathrm{R}=\mathrm{CHO}$<smiles>CC(=C[C@H](O)C(=O)C(C)=CCC1[C@H](C)[C@H]1C)CC=CC(C)(C)O</smiles>

agroskerin (150)

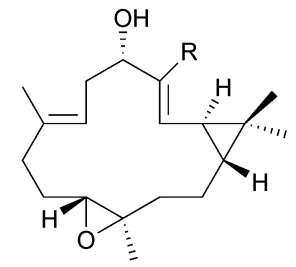

pekinenin $\mathrm{D}$ (153): $\mathrm{R}=\mathrm{CHO}$ pekinenin $\mathrm{E}$ (154): $\mathrm{R}=\mathrm{COOH}$

2.4.3 Other activities. Yuexiandajisu B (155) inhibited the proliferation of B lymphocytes. ${ }^{108}$ 


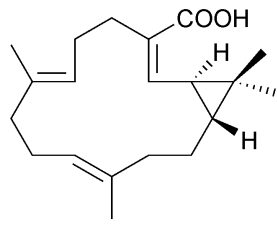

yuexiandajisu B

(155)

\subsection{Jatropholanes $\dagger$}

2.5.1 Antimicrobial activity. Sikkimenoid (156) demonstrated inhibitory activity against the microorganisms Staphylococcus aureus, Escherichia coli, Bacillus subtilis, Pseudomonas aeruginosa, and Staphylococcus epidermidis. ${ }^{\mathbf{1 1 6}}$

Malaria is one of the most common parasitic infections worldwide. Plasmodium falciparum, a protozoan parasite, is the most prevalent strain in Africa and also the most fatal.117 Jatropholone A (157) and compound 158 showed antiplasmodial activity against Plasmodium falciparum. ${ }^{118}$

sikkimenoid $\mathrm{B}$ (156)
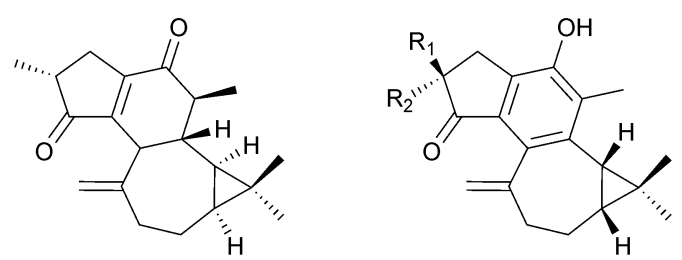

jatropholone $A$ (157): $R_{1}=M e, R_{2}=H$, 158: $\mathrm{R}_{1}=\mathrm{Me}, \mathrm{R}_{2}=\mathrm{OH}$ jatropholone $B(159): R_{1}=H, R_{2}=M e$, 160: $\mathrm{R}_{1}=\mathrm{OH}, \mathrm{R}_{2}=\mathrm{Me}$

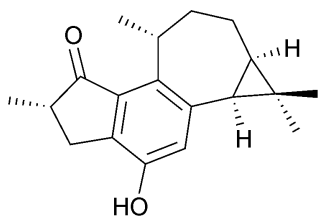

falodone

(161)

2.5.2 Cytotoxic activity. Jatropholones A (157) and B (159) exhibited antitumor activity. ${ }^{119,120}$ Compound 160 was mildly cytotoxic against Vero cells. ${ }^{\mathbf{1 2 1}}$ Moreover, falodone (161) strongly inhibited the proliferation of the A-549 human cancer cell line. ${ }^{122}$

2.5.3 Gastroprotective activity. Jatropholones A (157) and B (159) showed biological activity as gastroprotectors. Compound 157 was less active than compound 159 in preventing the appearance of gastric ulcers but both reduced gastric lesions. ${ }^{118}$

\subsection{Premyrsinanes $\uparrow$}

2.6.1 Modulation of multidrug resistance. Premyrsinanes 162-165, isolated from Euphorbia falcata, act with a mild to very strong synergy with doxorubicin against the MDR mouse lymphoma cell line. ${ }^{\mathbf{1 2 3 , 1 2 4}}$

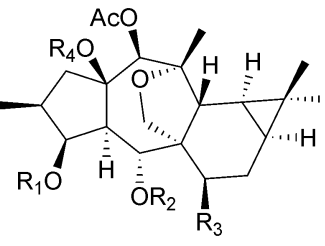

162: $\mathrm{R}_{1}=n$-hexanoyl, $\mathrm{R}_{2}=\mathrm{R}_{3}=\mathrm{R}_{4}=\mathrm{H}$ 163: $R_{1}=$ propanoyl, $R_{2}=A c, R_{3}=O B z, R_{4}=A c$ 164: $R_{1}=$ isobutanoyl, $R_{2}=H, R_{3}=O B z, R_{4}=A C$

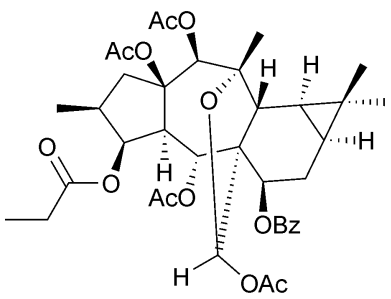

165

2.6.2 Neuroprotective activity. Euphorbiaproliferins I (166) and J (167) exhibited neuroprotective effects. ${ }^{\mathbf{1 2 5}}$

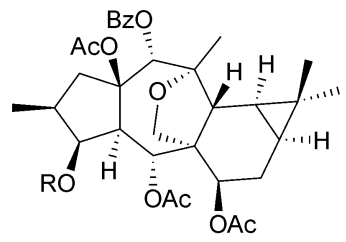

euphorbiaproliferin I (166):

$\mathrm{R}=$ propanoyl

euphorbiaproliferin $\mathrm{J}(167)$

$\mathrm{R}=$ butanoyl

\subsection{Euphoractine group $\dagger$}

Euphoractines are Natural Products that contain the rare 5-6-7-3 fused diterpene core obtained from a lathyrane skeleton by chemical cyclization. ${ }^{\mathbf{1 2 6}}$ The listed euphoractines showed no biological activities. ${ }^{127-130}$

\subsection{Other diterpenes $\dagger$}

Other compounds with a gem-dimethylcyclopropane in the structures have been isolated from many different sources. However, their structures do not belong to the above groups and were therefore placed together in this sub-group.

2.8.1 Antimicrobial activity. Riolozatrione (168) acts as an antibiotic against Staphylococcus aureus. ${ }^{131}$

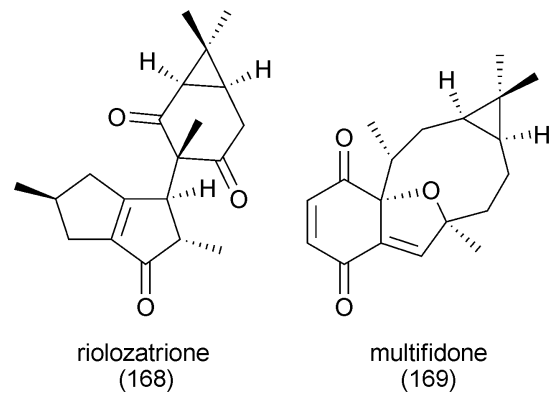

2.8.2 Cytotoxic activity. The cytotoxicity of multifidone (169) was measured against the cancerous cell lines TPH-1, HL60, A-375 and A-549. ${ }^{132}$

\section{Conclusions and future outlook}

In summary, this comprehensive review covered the isolation of diterpenes containing this gem-dimethylcarbocycle and whose biological activities are enhanced. The gem-dimethylcyclopropane 
ring remains intact in the biogenetic route leading to a large variety of secondary metabolites in many organisms. In fact, in these natural products the dimethylcyclopropyl unit remains as opposed to an isopropyl unit. This suggests the vital importance of this subunit in nature. Biological research on these gemdimethylcyclopropane derivatives has lent credence to the use of some plants in traditional medicine. Furthermore, the secondary metabolism of these plants and of a group of microorganisms, fungi or soft corals, revealed new activities for those compounds.

The study of the structure-activity relationships of these compounds has gained momentum in recent years, especially with the study of interactions with proteins kinase $\mathrm{C}$ and it is to be expected that this trend will continue in the years to come.

On the other hand, the massive genome sequencing projects of different types of organisms will lead to the discovery and identification of new cyclopropane-ring containing natural products and their biosynthetic gene clusters, which will help to unveil new terpene synthases and additional mechanisms for gem-dimethylcyclopropane ring formation.

We anticipate that this substantial number of diterpenoids containing the gem-dimethylcyclopropane ring could be used in the study of in vivo protein-NP interactions with the aim of designing new drugs. A deeper study of these biological interactions will reveal the role of this intriguing ring in the pathway of the modulation of diverse proteins kinase $\mathrm{C}$ and it will allow to modulate them. The occurrence of a wide range of diterpenoids with this feature or even derivatized products that have resulted in biological activities supports the biological potential of these structures. This is the main reason why they should deserve more attention. Therefore, these metabolites and those that will be isolated in the future as well as related compounds offer scope for development as potential therapeutic agents.

\section{Abbreviations}

$\begin{array}{ll}\text { 2-MeBu } & \text { 2-Methylbutanoyl } \\ \text { Ang } & \text { Angelate } \\ \text { Anth } & \text { Anthranoyl } \\ \text { Bz } & \text { Benzoyl } \\ \text { Cinn } & \text { Cinnamoyl } \\ \text { EBV } & \text { Epstein-Barr virus } \\ \text { HIV-1- } & \text { Human Immunodeficiency Virus-1-Long Terminal } \\ \text { LTR } & \text { Repeats } \\ \text { PKC } & \text { Protein Kinase C } \\ \text { PMA } & \text { Phorbol Myristate Acetate } \\ \text { Tig } & \text { Tigliate } \\ \text { TPA } & \text { Tetradecanoyl Phorbol Acetate }\end{array}$

\section{Acknowledgements}

This research was supported by grants from the Junta of Andalucía (PO7-FQM-02925). We thank Dr J. R. Hanson, Sussex University for manuscript revision.

\section{References}

1 B. R. Stockwell, Nature, 2004, 432, 846-854.

2 A. Vasas, D. Redei, D. Csupor, J. Molnar and J. Hohmann, Eur. J. Org. Chem., 2012, 5115-5130.

3 Q.-W. Shi, X.-H. Su and H. Kiyota, Chem. Rev., 2008, 108, 4295-4327.

4 W.-W. Tao, J.-A. Duan, Y.-P. Tang, N.-Y. Yang, J.-P. Li and Y.-F. Qian, Phytochemistry, 2013, 94, 249-253.

5 C. J. Thibodeaux, W. Chang and H. Liu, Chem. Rev., 2012, 112, 1681-1709.

6 M. T. Dueber, W. Adolf and C. A. West, Plant Physiol., 1978, 62, 598-603.

7 C. J. D. Mau and C. A. West, Proc. Natl. Acad. Sci. U. S. A., 1994, 91, 8497-501.

8 K.-X. Huang, Q.-L. Huang and A. I. Scott, Arch. Biochem. Biophys., 1998, 352, 144-152.

9 J. Kirby, M. Nishimoto, J. G. Park, S. T. Withers, F. Nowroozi, D. Behrendt, E. J. G Rutledge, J. L. Fortman, H. E. Johnson, J. V. Anderson and J. D. Keasling, Phytochemistry, 2010, 71, 1466-1473.

10 M. Wigler, D. DeFeo and I. B. Weinstein, Cancer Res., 1978, 38, 1434-1437.

11 V. Kinzel, J. Richards, K. Goerttler, H. Loehrke, G. Fürstenberger and F. Marks, IARC Sci. Publ., 1984, 56, 253-264.

12 I. B. Weinstein, L.-S. Lee, P. B. Fisher, A. Mufson and H. Yamasaki, J. Supramol. Struct., 1979, 12, 195-208.

13 D. T. Chu and D. K. Granner, J. Biol. Chem., 1986, 261, 16848-16853.

14 S. L. Poccote and R. W. Holz, J. Biol. Chem., 1986, 261, 18731877.

15 U. Testa, M. Titeux, F. Louache, P. Thomopoulos and H. Rochant, Cancer Res., 1984, 44, 4981-4986.

16 A. D. Kinghorn, J. Nat. Prod., 1979, 42, 112-115.

17 G. W. J. Olivier, M. G. Rowan, S. K. Branch, M. F. Mahon and K. C. Molloy, J. Chem. Soc., Perkin Trans. 1, 1992, 1831-1835.

18 P. P. Roux, B. A. Ballif, R. Anjum, S. P. Gygi and J. Blenis, Proc. Natl. Acad. Sci. U. S. A., 2004, 101, 13489-13494.

19 K. R. Gustafson, J. H. Cardellina, J. B. McMahon, R. J. Gulakowski, J. Ishitoya, Z. Szallasi, N. E. Lewin, P. M. Blumberg, O. S. Weislow and J. A. Beutler, J. Med. Chem., 1992, 35, 1978-1986.

20 J. Rullas, M. Bermejo, J. García-Pérez, M. Beltán, N. González, M. Hezareh, S. J. Brown and J. Alcamí, Antivir. Ther., 2004, 9, 545-554.

21 P. M. Blumberg and Z. Szallasi, PCT Int. Appl. WO 9427593 A1 19941208, 1994.

22 I. B. Baloch, M. K. Baloch and A. K. Baloch, Planta Med., 2010, 76, 809-814.

23 Q.-G. Ma, W.-Z. Liu, X.-Y. Wu, T.-X. Zhou and G.-W. Qin, Phytochemistry, 1997, 44, 663-666.

24 Y.-X. Sun and J.-C. Liu, Chem. Biodiversity, 2011, 8, 1205-1214.

25 J. Kulkosky, D. M. Culnan, J. Roman, G. Dornadula, M. Schnell, M. R. Boyd and R. J. Pomerantz, Blood, 2001, 98, 3006-3015. 
26 S. Bocklandt, P. M. Blumberg and D. H. Hamer, Antiviral Res., 2003, 59, 89-98.

27 G. Brooks, N. A. Morrice, C. Ellis, A. Aitken, A. T. Evans and F. J. Evans, Toxicon, 1987, 25, 1229-1233.

28 N. Márquez, M. A. Calzado, G. Sánchez-Duffhues, M. Pérez, A. Minassi, A. Pagani, G. Appendino, L. Diaz, M. A. MuñozFernández and E. Muñoz, Biochem. Pharmacol., 2008, 75, 1370-1380.

29 A. Pagani, C. Navarrete, B. L. Fiebich, E. Muñoz and G. Appendino, J. Nat. Prod., 2010, 73, 447-451.

30 L.-L. Pan, P.-L. Fang and X.-J. Zhang, J. Nat. Prod., 2011, 74, 1508-1512.

31 L. Ávila, M. Perez, G. Sanchez-Duffhues, R. HernándezGalán, E. Muñoz, F. Cabezas, W. Quinones, F. Torres and F. Echeverri, Phytochemistry, 2010, 71, 243-248.

32 N. Rudra, N. Singh and M. Gupta, J. Biosci., 1996, 21, 477-485. 33 I. B. Baloch, M. K. Baloch and Q. N. Saqib, Eur. J. Med. Chem., 2008, 43, 274-281.

34 M. O. Fatope, L. Zeng, J. E. Ohayaga, G. Shi and J. L. McLaughlin, J. Med. Chem., 1996, 39, 1005-1008.

35 S. M. Kupchan, I. Uchida, A. R. Branfman, R. G. Dailey Jr. and B. Y. Fei, Science, 1976, 191, 571-572.

36 Y.-B. Wang, P. Ji, H.-B. Wang and G.-W. Qin, Zhongguo Tianran Yaowu, 2010, 8, 94-96.

37 I. S. Aljancic, M. Pesic, S. M. Milosavjevic, N. M. Todorovic, M. Jadranin, G. Milosavljevic, D. Povrenovic, J. Bankovic, N. Tanic, I. D. Markovic, S. Ruzdijic, V. E. Vajs and V. V. Tesevic, J. Nat. Prod., 2011, 74, 1613-1620.

38 G. R. Pettit, S. Ducki, R. Tan, R. S. Gardella, J. B. McMahon, M. R. Boyd, G. R. Pettit III, P. M. Blumberg, N. E. Lewin, D. L. Doubek, L. P. Tackett and M. D. Williams, J. Nat. Prod., 2002, 65, 1262-1265.

39 L. Betancur-Galvis, E. Palomares, J. A. Marco and E. Estornell, J. Ethnopharmacol., 2003, 85, 279-282.

40 G. Goel, H. P. S. Makkar, G. Francis and K. Becker, Int. J. Toxicol., 2007, 26, 279-288.

41 W. Adolf and E. Hecker, Tetrahedron Lett., 1980, 21, 28872890.

42 L. Jing, Y. Fang, X. Ying, H. Wenxing, X. Meng, M. N. Syed and C. Fang, J. Appl. Entomol., 2005, 129, 98-104.

43 L. Jing, Y. Fang, X. Ying, H. Wenxing, X. Meng, M. N. Syed and C. Fang, Nongyaoxue Xuebao, 2005, 7, 29-34.

44 F. J. Evans, Toxicon, 1978, 16, 51-57.

45 G. Falsone and A. E. G. Crea, Liebigs Ann. Chem., 1979, 8, 1116-1121.

46 A. Marston and E. Hecker, Planta Med., 1984, 50, 319-322.

47 S. E. Taylor, M. A. Garfur, A. K. Choudhury and F. J. Evans, Experientia, 1981, 37, 681-682.

48 S. E. Taylor, M. Edwards, E. Williamson and F. J. Evans, J. Pharm. Pharmacol., 1981, 33, 54.

49 S. M. Zayed, M. Farghaly, S. M. Soliman, H. Gotta, B. Sorg and E. Hecker, J. Cancer Res. Clin., 2001, 127, 40-47.

50 S. Zayed, A. Hafez, W. Adolf and E. Hecker, Experientia, 1977, 33, 1554-1555.

51 A. E. Lockyer, J. N. Spinks, A. J. Walker, R. A. Kane, L. R. Noble, D. Rollinson, E. Dias-Neto and C. S. Jones, Dev. Comp. Immunol., 2007, 31, 763-782.
52 W. L. Lowe Jr., M. A. Yorek, C. W. Karpen, R. M. Teasdale, J. G. Hovis, B. Albrecht and C. Prokopiou, Mol. Endocrinol., 1992, 6, 741-752.

53 E. G. Puszkin and M. B. Zucker, Nature: New Biology, 1973, 245, 277-280.

54 L. G. Rao and T. M. Murray, Calcif. Tissue Int., 1989, 45, 354359.

55 C. Brodie and P. M. Blumberg, Apoptosis, 2003, 8, 19-27.

56 A. M. González-Guerrico, J. Meshki, L. Xiao, F. Benavides, C. J. Conti and M. G. Kazanietz, Biochem. Mol. Biol., 2005, 38, 639-645.

57 S. Oidovsambuu, C. Y. Kim, K. Kang, B. Dulamjav, T. Jigjidsuren and C. W. Nho, Planta Med., 2013, 79, 116122.

58 M. Bourjout, L. Delang, N. Van, H. Nguyen, J. Neyts, F. Guéritte, P. Leyssen and M. Litaudon, J. Nat. Prod., 2012, 75, 2183-2187.

59 O. O. Aiyelaagbe, K. Adesogan, O. Ekundayo and J. B. Gloer, Phytochemistry, 2007, 68, 2420-2425.

60 R. K. Devappa, H. P. S. Makkar and K. Becker, J. Am. Oil Chem. Soc., 2011, 88, 301-322.

61 G. Schmeda-Hirschmann, F. Tsichritzis and J. Jakupovic, Phytochemistry, 1992, 31, 1731-1735.

62 R. Pusztai, M.-J. U. Ferreira, N. Duarte, H. Engi and J. Molnar, Anticancer Res., 2007, 27, 201-206.

63 S. Budhiraja and A. P. Rice, Future Virol., 2013, 8, 649-659. 64 M. Daoubi, N. Marquez, N. Mazoir, A. Benharref, R. Hernández-Galán, E. Muñoz and I. G. Collado, Bioorg. Med. Chem., 2007, 15, 4577-4584.

65 H. Lage, N. Duarte, C. Coburger, A. Hilgeroth and M.-J. U. Ferreira, Phytomedicine, 2010, 17, 441-448.

66 I. B. Baloch, M. K. Baloch and Q. N. Saqib, Planta Med., 2006, 72, 830-834.

67 V. Ravikanth, V. L. N. Reddy, A. V. Reddy, K. Ravinder, T. P. Rao, T. S. Ram, K. A. Kumar, D. P. Vamanarao and Y. Venkateswarlu, Chem. Pharm. Bull., 2003, 51, 431-434.

68 S. B. Sanni, H. Behm, P. T. Beurskens, E. Y. Adesogan and J. I. Durodola, J. Chem. Crystallogr., 1988, 18, 575-582.

69 J.-Y. Zhang, C. Zhang, H.-B. Chen, L.-W. Fu, Y.-W. Tao, X.-Q. Zheng, Z.-M. Cao, Y.-F. Zhong and L.-H. Yu, J. Med. Plants Res., 2010, 4, 335-338.

70 N. Duarte, N. Gyemant, P. M. Abreu, J. Molnar and M.-J. U. Ferreira, Planta Med., 2006, 72, 162-168.

71 O. Wesolowska, J. Wisniewski, N. Duarte, M.-J. U. Ferreira and K. Michalak, Anticancer Res., 2007, 27, 4127-4134.

72 G. Appendino, C. Della Porta, G. Conseil, O. Sterner, E. Mercalli, C. Dumontet and A. Di Pietro, J. Nat. Prod., 2003, 66, 140-142.

73 C. Castro-González, R. Hernández-Galán, A. J. MacíasSánchez, F. Echeverri, N. Geribaldi-Doldan, A. AguileraDíaz, J. Dominguez-Riscart, A. Castillo-Domínguez and M. Murillo-Carretero, PCT Int. Appl., 2013, WO 2013057337 A2 20130425.

74 V. Ravikanth, V. L. N. Reddy, T. Prabhakar, P. V. Diwan, S. Ramakrishna and Y. Venkateswarlu, Phytochemistry, 2002, 59, 331-335. 
75 F. J. Miranda, J. A. Alabadi, M. Orti, J. M. Centeno, M. Pinon, A. Yuste, J. F. Sanz-Cervera, J. A. Marco and E. Alborch, J. Pharm. Pharmacol., 1998, 50, 237-241.

76 Y. Tian, W. Xu, C. Zhu, S. Lin, Y. Li, L. Xiong, S. Wang, L. Wang, Y. Yang, Y. Guo, H. Sun, X. Wang and J. Shi, J. Nat. Prod., 2011, 74, 1221-1229.

77 P. Hampson, K. Wang and J. M. Lord, Drugs Future, 2005, 30, 1003-1005.

78 S. A. Mason, S.-J. Cozzi, C. J. Pierce, S. J. Pavey, P. G. Parsons and G. M. Boyle, Invest. New Drugs, 2010, 28, 575-586.

79 W. Adolf and E. Hecker, Z. Krebsforsch. Klin. O., 1975, 84, 325-344.

80 Z.-Q. Lu, M. Yang, J.-Q. Zhang, G.-T. Chen, H.-L. Huang, S.-H. Guan, C. Ma, X. Liu and D.-A. Guo, Phytochemistry, 2008, 69, 812-819.

81 D. J. Pan, C. Q. Hu, J. J. Chang, T. T. Y. Lee, Y. P. Chen, H. Y. Hsu, D. R. McPhail, A. T. McPhail and K. H. Lee, Phytochemistry, 1991, 30, 1018-1020.

82 Y.-B. Wang, Y.-Y. Li, H.-B. Wang and G.-W. Qin, Zhongguo Tianran Yaowu, 2007, 5, 182-185.

83 T. S. Wu, Y. M. Lin, M. Haruna, D. J. Pan, T. Shingu, Y. P. Chen, H. Y. Hsu, T. Nakano and K. H. Lee, J. Nat. Prod., 1991, 54, 823-829.

84 L.-Y. Wang, N.-L. Wang, X.-S. Yao, S. Miyata and S. Kitanaka, Chem. Pharm. Bull., 2003, 51, 935-941.

85 L.-Y. Wang, N.-L. Wang, X.-S. Yao, S. Miyata and S. Kitanaka, J. Nat. Prod., 2002, 65, 1246-1251.

86 W. Adolf and E. Hecker, Z. Krebsforsch. Klin. O., 1975, 84, 325-344.

87 J. Hohmann, F. Evanics, L. Berta and T. Bartok, Planta Med., 2000, 66, 291-294.

88 A. R. Jassbi, Phytochemistry, 2006, 67, 1977-1984.

89 A. M. Rizk, F. M. Hammouda, M. M. El-Missiry, H. M. Radwan and F. J. Evans, Phytochemistry, 1985, 24, 1605-1606.

90 D. Uemura and Y. Hirata, Tetrahedron Lett., 1973, 11, 881-884.

91 W. Ye and R. W. Giblin-Davis, J. Parasitol., 2013, 99, 803815.

92 J. Shi, Z. Li, T. Nitoda, M. Izumi, H. Kanzaki, N. Baba, K. Kawazu and S. Nakajima, Z. Naturforsch., J. Biosci., 2008, 63, 59-65.

93 Q.-W. Shi, X.-H. Su and H. Kiyota, Chem. Rev., 2008, 108, 4295-4327.

94 J.-X. Shi, Z.-X. Li, T. Nitoda, M. Izumi, H. Kanzaki, N. Baba, K. Kawazu and S. Nakajima, Biosci., Biotechnol., Biochem., 2007, 71, 1086-1089.

95 I. B. Baloch, M. K. Baloch and A. K. Baloch, Eur. J. Med. Chem., 2009, 44, 3188-3194.

96 B. A. Nascimento and C. L. Zani, Phytochem. Anal., 1999, 10, 93-96.

97 C. L. Zani, A. Marston, M. Hamburger and K. Hostettmann, Phytochemistry, 1993, 34, 89-95.

98 Q. L. Dang, Y. H. Choi, G. J. Choi, K. S. Jang, M. S. Park, N.-J. Park, C. H. Lira, H. Kim, L. H. Ngoc and J.-C. Kim, J. Asia Pacific Entomol., 2010, 13, 51-54.

99 M. Hirota, H. Ohigashi, Y. Oki and K. Koshimizu, Agric. Biol. Chem., 1980, 44, 1351-1356.
100 I. B. Baloch, M. K. Baloch and Z. N. Saqib, Helv. Chim. Acta, 2005, 88, 3145-3150.

101 R. R. Upadhyay, M. Ansarin, M. H. Zarintan and P. Shakui, Experientia, 1976, 32, 1196-1197.

102 W. Zheng, Zhongcaoyao, 2004, 35, 65-68.

103 S. Nunomura, S. Kitanaka and C. Ra, Biol. Pharm. Bull., 2006, 29, 286-290.

104 X.-L. Li, Y. Li, S.-F. Wang, Y.-L. Zhao, K.-C. Liu, X.-M. Wang and Y.-P. Yang, J. Nat. Prod., 2009, 72, 1001-1005.

105 Y. Bai, Y.-P. Yang and Y. Ye, Tetrahedron Lett., 2006, 47, 6637-6640.

106 H. Chen and Z. J. Jia, Indian J. Chem. Sect. B: Org. Chem. Incl. Med. Chem., 1996, 35B, 1308-1310.

107 Y.-H. Choi, J. M. Pezzuto, A. D. Kinghorn and N. R. Farnsworth, J. Nat. Prod., 1988, 51, 110-116.

108 Y. Kashman, M. W. Bernart, M. Tischler, J. H. Cardellina and M. R. Boyd, J. Nat. Prod., 1994, 57, 426-430.

109 Z.-H. Xu, J. Sun, R.-S. Xu and G.-W. Qin, Phytochemistry, 1998, 49, 149-151.

110 Y. Li, M. Carbone, R. M. Vitale, P. Amodeo, F. Castelluccio, G. Sicilia, E. Mollo, M. Nappo, G. Cimino, Y. W. Guo and M. Gavagnin, J. Nat. Prod., 2010, 73, 133-138.

111 V. A. Carneiro, H. Silva dos Santos, F. V. S. Arruda, P. N. Bandeira, M. R. J. R. Albuquerque, M. O. Pereira, M. Henriques, B. S. Cavada and E. H. Teixeira, Molecules, 2011, 16, 190-201.

112 Y. H. Choi, J. Kim, J. M. Pezzuto, A. D. Kinghorn, N. R. Farnsworth, H. Lotter and H. Wagner, Tetrahedron Lett., 1986, 27, 5795-5798.

113 Q.-L. Liang, C.-C. Dai, J.-H. Jiang, Y.-P. Tang and J.-A. Duan, Fitoterapia, 2009, 80, 514-517.

114 F.-G. Shao, R. Bu, C. Zhang, C.-J. Chen, J. Huang and J.-H. Wang, J. Asian Nat. Prod. Res., 2011, 13, 805810.

115 C.-X. Zhang, S.-J. Yan, G.-W. Zhang, W.-G. Lu, J.-Y. Su, L.-M. Zeng, L.-Q. Gu, X.-P. Yang and Y.-J. Lian, J. Nat. Prod., 2005, 68, 1087-1089.

116 Y.-F. Yang, J.-Q. Liu, L. Shi, Z.-R. Li and M.-H. Qiu, Nat. Prod. Bioprospect., 2013, 3, 99-102.

117 S. A. Pawluk, K. J. Wilby and M. H. H. Ensom, Clinical Pharmacokinetics, 2013, 52, 153-167.

118 S. Sutthivaiyakit, W. Mongkolvisut, S. Prabpai and P. Kongsaeree, J. Nat. Prod., 2009, 72, 2024-2027.

119 M. Pertino, G. Schmeda-Hirschmann, J. A. Rodriguez and C. Theoduloz, Planta Med., 2007, 73, 1095-1100.

120 K. K. Purushothaman, S. Chandrasekharan, A. F. Cameron, J. D. Connolly, C. Labbe, A. Maltz and D. S. Rycroft, Tetrahedron Lett., 1979, 20, 979-980.

121 S. Sutthivaiyakit, W. Mongkolvisut, S. Prabpai and P. Kongsaeree, J. Nat. Prod., 2009, 72, 2024-2027.

122 A. Falodun, Q. Sheng-Xiang, G. Parkinson and S. Gibbons, Pharm. Chem. J., 2012, 45, 636-639.

123 E. Sulyok, A. Vasas, D. Redei, P. Forgo, Z. Kele, G. Pinke and J. Hohmann, Tetrahedron, 2011, 67, 7289-7293.

124 A. Vasas, E. Sulyok, A. Martins, D. Redei, P. Forgo, Z. Kele, I. Zupko, J. Molnar, G. Pinke and J. Hohmann, Tetrahedron, 2012, 68, 1280-1285. 
125 J. Xu, Y. Guo, C. Xie, Y. Li, J. Gao, T. Zhang, W. Hou, L. Fang and L. Gui, J. Nat. Prod., 2011, 74, 2224-2230.

126 W. Adolf and E. Hecker, Tetrahedron Lett., 1970, 22412244.

127 J. Shi and Z. Jia, Phytochemistry, 1995, 38, 1445-1447.

128 L. Barboni, P. Gariboldi, E. Torregiani, G. Appendino, B. Gabetta, G. Zini and E. Bombardelli, Phytochemistry, 1993, 33, 1553.
129 J. Shi, Z. Jia and L. Yang, Phytochemistry, 1992, 32, 208-210. 130 A. Vasas, J. Hohmann, P. Forgo and P. Szabo, Tetrahedron, 2004, 60, 5025-5030.

131 W. H. Watson, V. Zabel and X. A. Dominguez, Acta Crystallogr., Sect. B: Struct. Sci., 1982, B38, 689-691.

132 B. Das, K. R. Reddy, B. Ravikanth, T. V. Raju, B. Sridhar, P. U. Khan and T. V. Rao, Bioorg. Med. Chem. Lett., 2009, 19, 77-79. 